\title{
Comparación entre dos métodos de estimación de edad del venado de cola blanca (Odocoileus virginianus) con fines de manejo cinegético
}

\author{
A comparison between two aging methods of \\ white-tailed deer (Odocoileus virginianus) for hunting purposes
}

\author{
Diego Olivera Vásquez ${ }^{1}$ y Pedro Vásquez Ruesta²
}

\begin{abstract}
Resumen
Se estimó la edad de 298 venados abatidos en distintas temporadas de caza en el Coto de Caza El Angolo, Piura, Perú, mediante el método de desgaste y erupción de las piezas dentales. Luego se tomó una muestra de 82 venados y se analizó con el método de conteo de los anillos dentales. Se utilizó la muestra para comparar los resultados de ambas metodologías. Adicionalmente se utilizó una pequeña muestra de mandíbulas de animales de edad conocida para comparar el ajuste de ambas metodologías a la edad real de los animales. No existieron diferencias significativas entre los resultados de ambos métodos, ni tampoco entre la edad real y las estimaciones etarias. Se concluyó que el método de desgaste y erupción de las piezas dentales es el método más recomendable para un caso como el estudiado.
\end{abstract}

Palabras clave: Odocoileus virginianus; venado de cola blanca; estimación de edad; anillos dentales; método de desgaste; manejo de fauna; Coto de Caza El Angolo.

\begin{abstract}
The ages of 298 deer taken during different hunting seasons in El Angolo Hunting Reserve, Piura, Peru, were estimated according to the use and wear method. Then, a sample of 82 mandibles was taken and was analyzed with the annuli count method. The sample was then used to compare the results of both techniques. Additionally, a small sample of known aged mandibles was used to compare the adjustment of both methods to the real age of the deer. There were no significant differences neither between both techniques, nor between the real age and the age estimations. It was decided that the use and wear method is the most recommendable method for a case like the one studied.
\end{abstract}

Key words: Odocoileus virginianus; white-tailed deer; aging; dental annuli; use and wear method; wildlife management; El Angolo Hunting Reserve.

\footnotetext{
${ }^{1}$ Consultor independiente, Alemania. E-mail: diego.olivera@tum.de

${ }^{2}$ Facultad de Ciencias Forestales, Universidad Nacional Agraria La Molina, Perú.
} 


\section{Introducción}

El manejo cinegético de fauna silvestre requiere de información y conocimiento para cumplir con los objetivos de determinadas áreas. En el caso de las poblaciones de venados de cola blanca (Odocoileus virginianus) del Coto de Caza de Caza El Angolo (CCEA), dicho manejo se basa en la experiencia que han ido adquiriendo las dos instituciones encargadas del mismo: el Club de Caza, Pesca y Turismo Piura (CCPTP) y la Universidad Nacional Agraria La Molina (UNALM). Entre otros componentes, se analiza la edad de los individuos cobrados para poder asegurar que el promedio anual sobrepasa un límite preestablecido. Actualmente se estima la edad utilizando el método de desgaste y erupción de las piezas dentales (Severinghaus 1949), el cual cuenta con usuarios en todo el mundo, pero lamentablemente no existe un trabajo para el Perú que lo respalde. Por este motivo, se vuelve necesario un estudio que lo contraste con otra metodología actualmente utilizada y que haya demostrado funcionar adecuadamente como el método de conteo de los anillos dentales (Gilbert 1966, Erickson et al. 1970, Lockard 1972, Hamlin et al. 2000).

Severinghaus (1949) desarrolló el método de desgaste y erupción de las piezas dentales con venados de cola blanca (Odocoileus virginianus). Realizó mediciones desde las encías hasta las cúspides de los dientes para ver cómo éstos se iban desgastando y presentó fotografías que representaban adecuadamente a cada clase de edad. Posteriormente, Taber (1960, 1971) refirió información similar como técnica recomendada para estimar la edad de dichos venados y de otros cérvidos, y además añadió tablas de erupción de los dientes y dibujos de las mandíbulas mostrando el desgaste por cada clase de edad. Prueba de la aceptación de este método, se comienza a producir un producto comercial: el Speedager (Wildlife Heritage Productions 1984). Este producto consiste en una cartilla con fotografías y texto explicativo de cada clase de edad. Las mandíbulas a ser evaluadas, deben observarse minuciosamente y deben ser comparadas con cada una de las clases presentes en la cartilla, decidiendo luego a qué clase de edad pertenecen. Las clases de edad presentes en dicha cartilla son: cría, 1,5 años, 2,5 años, 3,5 años, 4,5 años, 5,5 años y $6,5+$ años. En este trabajo son denominadas 0 , I, II, III, IV, V y VI+ respectivamente.

El método recibió luego buenas críticas para distintos tipos de ciervos (Larson y Taber 1987) y continuó siendo utilizado por la facilidad de aplicación y la calidad de los resultados obtenidos (Kroll 1994). Sin embargo, existió discusión sobre la precisión y la tasa de desgaste (Low y Cowan 1963, Gilbert y Stolt 1970, Hamlin et al. 2000), lo que llevó a recomendar la búsqueda de un método más general y preciso (Gilbert 1966).

Basados en los trabajos de Scheffer (1950) y Laws (1953) (citados por Low y Cowan 1963), otros autores empezaron a desarrollar un nuevo método de estimación de edad para cérvidos. El método consistió, en líneas generales, en cortar secciones muy delgadas de la raíz de los dientes, que luego al ser colocadas al microscopio permiten la visualización y el conteo de los anillos de crecimiento. Los anillos se depositan anualmente en el cemento dental y se presentan en bandas claras y oscuras alternas que representan los períodos de abundancia y escasez de alimentos como primavera e invierno del hemisferio norte respectivamente (Sergeant y Pimlott 1959, Low y Cowan 1963, Gilbert 1966).

Otros autores lograron buenos resultados con el uso de esta metodología, a pesar de las dificultades encontradas al trabajar y al visualizar los anillos (Brokx 1972). Se encontraron anillos presentes en venados de cola blanca $(O$ virginianus), de cola negra (O. hemionus) e incluso en renos (Rangifer tarandus), los cuales demostraron completa o alta concordancia con la edad real de los animales (Reimers y Nordby 1968, Erickson y Seliger 1969, Lockard 1972, Hamlin et al. 2000).

Por el contrario, trabajos en regiones cálidas, así como en lugares con inviernos poco severos y con poca estacionalidad (Connolly et al. 1969, Gasaway et al. 1978, Cook y Hart 1979, Hacket et al. 1979, Rice 1980) generaron 
discusión y recomendaron el uso conjunto de ambas metodologías (Jacobson y Reiner 1989). Maffei y Becerra (2000), al trabajar con ungulados tropicales, sobrepasaron las dificultades encontradas para la visualización de los anillos. Sin embargo, concluyeron que dada la necesidad de más recursos para aplicar el método de conteo de anillos, el método de desgaste es la alternativa de elección, siempre y cuando se encuentre bien calibrado.

El objetivo del presente estudio es determinar, mediante una comparación entre dos métodos de estimación de edad para cérvidos, el más conveniente para un caso como el del CCEA. Para esto se tendrá en cuenta que el requerimiento del usuario (CCPTP) es poder contar con un método que otorgue la oportunidad de asignar edades de manera rápida, práctica, a un costo accesible y de manera individual a cada mandíbula, para luego obtener un promedio por temporada.

\section{Materiales y Métodos}

\section{El área de estudio}

El Coto de Caza El Angolo está ubicado en la costa norte del Perú en el departamento de Piura, provincias de Sullana y Talara $\left(4^{\circ} 11^{\prime}\right.$ a $4^{\circ} 26^{\prime} 37^{\prime \prime}$ S y $80^{\circ} 37^{\prime} 57^{\prime \prime}$ a $80^{\circ} 56^{\prime} 45^{\prime \prime}$ O). El rango altitudinal se encuentra entre 500 y 1600 msnm aproximadamente. La extensión oficial es de 65000 ha. El Sector Sauce Grande, lugar del cual provienen las mandíbulas de este trabajo, es administrado mediante un contrato de administración parcial por el CCPTP. Está ubicado en el extremo sur este del coto, tiene una extensión de 9918 ha (Vásquez et al. 2007).

El Angolo se encuentra en la provincia biogeográfica del Bosque Seco Ecuatorial (Udvardy 1975, citado por Vásquez et al. 2007). Predominan los árboles deciduos y el material arbustivo, además de las plantas espinosas y las cactáceas columnares. El contraste entre la época seca y la época de lluvias es muy notorio, cambiando la expresión del bosque. Con las lluvias los árboles deciduos se cubren de hojas, las quebradas se llenan de agua y las herbáceas fácilmente sobrepasan el metro de altura.
Además, el área del CCEA está fuertemente influenciada por la corriente de Humboldt, la corriente del Niño, la Cordillera de los Andes y el Ecuador; su ubicación geográfica hace que existan interacciones de componentes atmosféricos tanto continentales como marinos. Las precipitaciones son muy variables incluso dentro del área perteneciente al contrato de administración, registrando un promedio anual de 483,8 mm para la estación meteorológica instalada en Sauce Grande. Los suelos predominantes son vertisoles y yermosoles, siendo los primeros los que sostienen mayormente la biomasa de los bosques (Vásquez et al. 2007).

\section{Metodología}

Se siguieron tres fases para la elaboración del estudio:

- Método de desgaste y erupción de las piezas dentales: Primero se acondicionaron las mandíbulas que se hallaban almacenadas en el Laboratorio de Fauna Silvestre de la Facultad de Ciencias Forestales - Universidad Nacional Agraria La Molina (Lima, Perú). Estas mandíbulas fueron colectadas durante las temporadas de caza desde 1996 hasta 2009. Se contaba con un total de 306.

Luego se utilizó la cartilla de determinación de edad Speedager para asignar edades a la totalidad de las mandíbulas. Cada mandíbula fue comparada detenidamente con las fotografías y descripciones presentes en la cartilla para determinar a qué categoría pertenecían. Luego de clasificadas las mandíbulas se generó una base de datos con la información. Las clases utilizadas fueron las definidas en el Speedager: 0, I, II, III, IV, V y VI+.

Adicionalmente, se realizó una prueba denominada "Prueba de consistencia del desgaste", cuyo fin fue confirmar que trabajando de acuerdo a las instrucciones, independientemente de la frecuencia y sin importar cuantas veces se le asigne una categoría de edad a una mandíbula, las estimaciones son suficientemente similares para considerar al método como consistente. La prueba consistió en tomar una muestra de aproximadamente el 30\% del total de mandíbulas y asignarles 
repetidamente una edad, dejando pasar entre una y dos semanas por vez. Luego se aplicó una prueba de Chi-cuadrado para determinar qué tan similares fueron las distintas fechas de evaluación entre sí.

- Método de conteo de los anillos dentales de crecimiento: Se realizó un muestreo por limitaciones presupuestales. Una vez determinado el tamaño de muestra y seleccionadas las mandíbulas al azar, se extrajeron los dientes incisivos de las mismas. Para esto, las mandíbulas se remojaron durante una noche en agua de manera que los tejidos se suavizaran. Luego, utilizando una navaja, se realizó un corte entre los dientes deseados y cortes adicionales al lado de cada uno en caso fuera necesario. Finalmente se utilizó un alicate para palanquear los dientes y extraerlos. Los dientes fueron colocados en sobres de papel, los cuales fueron rotulados con el mismo código que la mandíbula de la cual provenían.

Los dientes fueron entregados al Laboratorio de Histología y Patología de la Facultad de Veterinaria y Zootecnia de la Universidad Peruana Cayetano Heredia (UPCH), lugar donde fueron preparadas las láminas histológicas necesarias para el estudio. Los dientes fueron fijados en formol $10 \%$ durante $48 \mathrm{~h}$. Luego se colocaron en ácido nítrico 5\% disuelto en etanol $80 \%$ para la descalcificación. Este proceso duró una semana aproximadamente. La solución fue renovada cada $48 \mathrm{~h}$ y se mantuvo en constante agitación para mejor acción del ácido. A continuación se deshidrataron los dientes en etanol a distintas concentraciones, para luego ser transferidos a un substituto comercial del xilol (NeoClear ${ }^{\varpi}$ ), el cual es miscible con el alcohol y con el medio de inclusión (parafina líquida). Los dientes se impregnaron de parafina líquida para que esta rodeara y penetrara al tejido formando un bloque sólido. Una vez impregnados los dientes de parafina, se colocaron en moldes $\mathrm{y}$ fueron llevados a la refrigeradora. El taco formado fue cortado con un micrótomo en secciones transversales de $10 \mu \mathrm{m}$ de grosor para permitir el paso de la luz. Finalmente fueron coloreados con Hematoxilina de Harris durante $10 \mathrm{~min}$, nuevamente deshidratados y montados con Entellan ${ }^{\circledR}$ en un portaobjetos.
Las láminas listas fueron analizadas al microscopio. Para esto se utilizaron las instalaciones del Laboratorio de Anatomía de la Madera de la Facultad de Ciencias Forestales - UNALM y también las instalaciones del Laboratorio de Histología y Patología de la Facultad de Veterinaria y Zootecnia - UPCH. Las láminas fueron visualizadas con 100x, 200x y 400x cuando fue necesario. El proceso consistió en ubicar una porción del borde del diente en donde se visualizaran claramente los anillos de crecimiento, para luego contarlos y proceder con las capturas digitales. Se siguió la recomendación de Brokx (1972) acerca de contar el máximo número de anillos visibles, pero también se decidió que para los casos en que no se tuviera total seguridad de que se alcanzaba cierta clase, se optaría por permanecer en la clase previa. Las edades tomaron los valores 0, I, II, III, IV, V y VI+, igual que en el método anterior y una vez clasificados todos los dientes de la muestra, fueron almacenados en una base de datos.

- Comparación entre los resultados de la asignación mediante el método de desgaste y el método de anillos: Se aplicaron dos pruebas estadísticas. Primero se aplicó la prueba de Wilcoxon utilizando los datos pareados obtenido del muestreo. Por cada individuo muestreado, existió una estimación con el método de desgaste y una con el método de conteo de anillos. Se utilizaron dichos pares de datos para probar si ambos métodos podían ofrecer resultados similares. El nivel de significancia elegido fue de $5 \%$ y la prueba fue de dos colas porque no establecía ninguna dirección en la hipótesis nula. La prueba fue aplicada con el software SPSS 15.0.

La segunda prueba fue la de Friedman. Para esto, se utilizó una pequeña muestra de mandíbulas provenientes de animales de edad conocida de procedencia distinta a las anteriores. Éstas fueron analizadas con ambas metodologías estudiadas, sin conocer la edad real, ni la edad asignada mediante el otro método. Luego se utilizó dicha muestra para probar si existieron diferencias significativas entre la edad real de los animales, la edad estimada 
mediante el método de desgaste y la edad estimada mediante el método de conteo de anillos. $\mathrm{Al}$ igual que en el caso anterior, la prueba se realizó con $\alpha=5 \%$ y fue aplicada con SPSS 15.0.

\section{Resultados}

\section{Método de desgaste y erupción de las piezas dentales}

Se acondicionaron 306 mandíbulas, de las cuales ocho resultaron inservibles para el estudio y fueron descartadas. El tamaño de la población quedó en 298 mandíbulas.

Las mandíbulas se distribuyeron en las clases 0 - V (Cuadro 1). La mayoría de venados cazados se ubicaron en las clases II y III, es decir que al momento de ser cazados tenían entre dos y cuatro años. Las clases de los extremos fueron las que menos individuos presentaron. El promedio etario resultó 2,89 años (clase II).

La prueba de consistencia entre las distintas fechas de evaluación no presentó diferencias significativas $\left(x^{2}=13.64, p=0.6249\right)$, indicando similitud entre las fechas de evaluación. La mayoría de observaciones se concentraron en las clases II y III. La Figura 1 ilustra las frecuencias de las distintas fechas de evaluación y de las frecuencias esperadas utilizadas para la prueba. Las clases 0 y I fueron agrupadas para cumplir con los requerimientos de la prueba (Siegel y Castellan 1988).

\section{Método de conteo de los anillos dentales de crecimiento}

El tamaño de muestra fue de 95 dientes, pero luego de la elaboración de las láminas histológicas, se eliminaron algunas por resultar inservibles, resultando el tamaño final de muestra en 82.

Durante la preparación de láminas, la orientación elegida fue la transversal puesto que se podían realizar más cortes que abarcaban toda la circunferencia del diente. Además, dicho plano podía brindar mejor resolución en las capas de cemento (Gasaway et al. 1978, Avadhani et al. 2009).

La distribución de los dientes se observa en el Cuadro 2. Existó una acumulación en las clases I, II y III (entre uno y cuatro años). Las otras clases se encontraron pobremente representadas. El promedio resultó 2,79 años (clase II).

\section{Comparación entre los resultados de la asig- nación mediante el método de desgaste y el método de los anillos}

No existieron diferencias significativas entre los resultados arrojados por ambos métodos (Wilcoxon: $z=-1.939, p=0.053$ ). De los

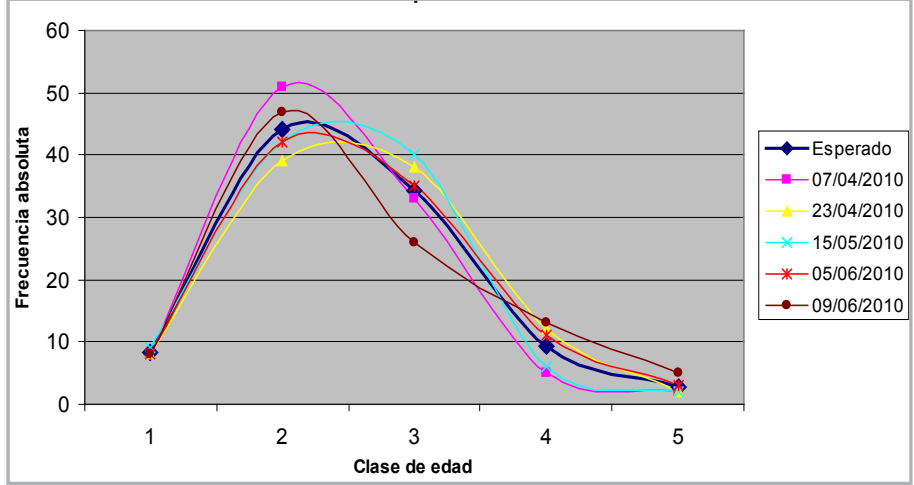

Figura 1. Curvas de frecuencias para distintas fechas de medición.

\begin{tabular}{|l|c|c|c|c|c|c|c|}
\hline Clase de edad & 0 & I & II & III & IV & V & Total \\
\hline $\mathbf{N}^{\circ}$ de mandíbulas & 5 & 21 & 160 & 80 & 26 & 6 & 298 \\
\hline
\end{tabular}

Cuadro 1. Distribución de mandíbulas por clase de edad $(0-\mathrm{V})$ según el método de desgaste y erupción de las piezas dentales. 


\begin{tabular}{|l|c|c|c|c|c|c|c|}
\hline Clase de edad & 0 & I & II & III & IV & V & Total \\
\hline $\mathbf{N}^{0}$ de mandíbulas & 2 & 24 & 23 & 23 & 6 & 4 & 82 \\
\hline
\end{tabular}

Cuadro 2. Distribución de mandíbulas por clase de edad (0 - V) según el método conteo de los anillos dentales de crecimiento.

82 casos, hubo 21 coincidencias que la prueba no tomó en cuenta para la comparación, analizando finalmente 61 pares de datos. La prueba indicó similitud entre ambos métodos.

Tampoco existieron diferencias significativas entre ninguna de las tres medidas existentes por cada individuo (Friedman: $x_{r}^{2}=0.0, p=$ 1.00, $n=7$ ). Ambos métodos estudiados se ajustaron correctamente a la edad real de los animales, indicando que su uso es equivalente.

\section{Discusión}

Una consideración importante a tener en cuenta durante la discusión, es recordar lo que significa manejo cinegético: existe caza deportiva y el usuario final del recurso es el cazador. Éste deseará abatir a un animal de buen porte, con una cornamenta atractiva y que supere los límites reglamentarios definidos por el plan de manejo del área en cuestión.

Ambos métodos cumplieron con asignar una clase de edad a las mandíbulas de los venados en las que fueron usados y presentaron similitudes y diferencias. Los resultados de las dos primeras fases brindaron una descripción de los datos y permitieron identificar qué clases etarias se encuentran más o menos representadas según cada método.

La clase I no estuvo bien representada con el método de desgaste, lo cual es de esperarse debido a la baja probabilidad de que un individuo de esa clase alcance una cornamenta atractiva para un cazador. Por el contrario, en el método de los anillos, la clase I muestra valores relativos similares a las dos clases siguientes. El método de desgaste agrupa a más del 50\% de individuos analizados en la clase II, mientras que el de anillos sólo supera levemente la mitad del valor anterior. Las clases III, IV y V mantienen la misma abundancia relativa con ambos métodos. Por otro lado, existieron diferencias en términos de distribución. Mientras que el mét- odo de desgaste proporcionó valores distintos para cada clase etaria (Cuadro 1), el de anillos parece agrupar los resultados en dos grandes grupos (Cuadro 2): los menores (clases I, II y II) y los mayores (clases IV y V). Sin embargo, este hecho por sí solo, no es evidencia de que un método sea mejor que el otro. A pesar de las diferencias en las clases I y II y de la distribución, los promedios etarios para ambos métodos presentan valores cercanos y superan el valor promedio de 2,5 años, recomendado para manejo cinegético de calidad (Adams 2004).

El hecho mencionado sobre la cornamenta de los venados y las recomendaciones para manejo cinegético, se basan en la biología del venado de cola blanca. Durante el primer año (clase 0) los animales desarrollan botones. Posteriormente, pequeños "cachitos" (spikes) o astas que van desarrollándose hasta donde la carga genética lo permita, de acuerdo a la calidad del alimento y la edad (Halls 1980, Sauer 1984).

La prueba de consistencia permitió comprobar la objetividad del método de desgaste. Un paso no incluido en los resultados, mostró que la variabilidad entre fechas de estimación aumentaba con la clase de edad. Este hecho sugeriría subjetividad. Sin embargo, los resultados de esta prueba brindaron suficiente sustento estadístico para rechazar tal posibilidad. La Figura 1 permite apreciar cómo las curvas de frecuencia llegan incluso a traslaparse, reafirmando la similitud entre las mismas.

\begin{tabular}{|l|l|l|}
\hline Categoría & Desgaste & Anillos \\
\hline Entrenamiento requerido & Medio & Medio-alto \\
\hline Facilidad & Alta & Poca \\
\hline Equipamiento necesario & Bajo & Alto \\
\hline Tiempo requerido & Corto & Extenso \\
\hline Costo & Bajo & Medio-alto \\
\hline
\end{tabular}

Cuadro 3. Características más importantes a considerar para el empleo de los dos métodos. 
La tercera fase permitió probar el ajuste entre ambos métodos y también entre los dos métodos y la edad real de los individuos. Si bien la prueba de Wilcoxon confirma la similitud entre el método de desgaste y el de anillos, no toma en cuenta las coincidencias entre pares. El hecho de existir 21 casos en los que la clase de edad asignada fue la misma, sugiere similitudes adicionales a las que se interpretan de la prueba. Lamentablemente éstas no pueden ser cuantificadas.

La comparación entre los individuos de edad conocida y las estimaciones por ambos métodos tampoco halló diferencias significativas. Esto indica que los dos métodos estudiados se ajustan correctamente a la edad real de los animales. Al igual que en la prueba anterior, no se percibió una tendencia a sub o sobre estimaciones, por lo tanto el uso es equivalente y la elección de tal o cual dependerá de criterios adicionales.

El Cuadro 3 ofrece factores a considerar que pueden ayudar a una toma de decisión y se comentan brevemente. El entrenamiento requerido y la facilidad de aplicación se desprenden de los antecedentes y la metodología. El método de desgaste resultó más sencillo y más rápido que el de anillos. Con el primero no es posible asignar edades a venados mayores de la clase $\mathrm{VI}+$, traduciéndose en una desventaja contra el métodos de los anillos. Este último ha proporcionado precisión incluso en animales de edad avanzada (19,5 años) (Thomas y Bandy 1973). Mientras que el método de desgaste requirió práctica y fue aplicado directamente por los autores, el de anillos tuvo que ser parcialmente tercerizado. De aquí también se desprende que mientras el primero requiere de un espacio para trabajar y una cartilla de identificación, el segundo requiere equipos de laboratorio.

A pesar de existir evidencia previa de la mejor zona (Low y Cowan 1963, Thomas y Bandy 1973) y de la orientación de corte (Gasaway et al. 1978, Avadhani et al. 2009) para el método de los anillos, existieron dificultades inherentes al mismo. La literatura ha atribuido estas dificultades a factores medioambientales (Lockard 1972, Brokx 1972) y al cuidado al momento de preparación de las láminas histológicas (Roy Andrade, comunicación personal). Además, el conteo de los anillos se dificultó en algunos casos debido a la cercanía entre las líneas existentes y los múltiples cortes que debieron practicarse a fin de buscar el mejor.

La cantidad de actividades necesarias para aplicar uno u otro método y el costo que éstas involucran, son factores fundamentales. Para utilizar el método de los anillos se incurre en un mayor número de actividades desde el momento en que se caza el animal. Esto se traduce en una diferencia de costo unitario de al menos a S/. 25 (aprox. US\$ 9.00, T/C en diciembre de 2011), lo cual dependiendo de la cantidad de animales que se esté extrayendo, puede ser un costo considerable.

El bajo costo, el poco tiempo y equipamiento requerido y la facilidad de aplicación que ofrece el método de desgaste, son características que lo distinguen del método de conteo de anillos. Considerando también los resultados obtenido de las comparaciones, el método de desgaste y erupción de las piezas dentales resulta favorecido.

Finalmente, es fundamental considerar la necesidad de manejo existente. Para el caso estudiado, los animales provienen de una población en estado natural de la que se extrae regularmente una parte. El requerimiento es poder contar con un método práctico y confiable que permita hallar un promedio. Basta con lograr un buen acercamiento, mas no es fundamental tener total precisión de cuántos meses y años tenía tal o cual animal al momento de su extracción. La valla que deben superar los animales y que el método a utilizar debe ser capaz de elegir, la define el usuario. Mientras un método cumpla, entonces será el adecuado.

\section{Conclusiones}

El método de desgaste y erupción de las piezas dentales se desarrolla con menor costo, menor tiempo y mayor facilidad.

El método de conteo de anillos dentales tiene un costo mayor que el de desgaste, requiere más tiempo y equipos, y presenta dificultades en la lectura de los anillos. 
La experiencia y el uso constante de un patrón para comparación son elementos fundamentales para la estimación de edad mediante el método de desgaste y erupción de las piezas dentales.

Realizar más de una repetición al trabajar con el método de desgaste y erupción de las piezas dentales brinda la oportunidad de reducir los errores involuntarios que puedan presentarse (cansancio, ausencia de luz, etc.).

Ambos métodos cumplen con asignar una edad a las mandíbulas de los venados, pero el método de desgaste no asigna más allá de la clase VI+, mientras que el de los anillos sí lo permite. Su uso, es recomendable para determinadas situaciones.

Ambos métodos arrojaron resultados similares y mostraron un ajuste correcto a los animales de edad conocida. Dados los resultados equivalentes y las diferencias en cuanto a los requerimientos y los costos, el método de desgaste y erupción de las piezas dentales es la elección para un caso como el del Coto de Caza El Angolo.

\section{Bibliografía}

Adams, K. 2004. Deer management strategies. Quality Deer Management Association QDMA. http://www.qdma.com/what-we-do/ articles/deer-biologymanagement/deer-management-strategies/ Consultado 26 de Sept 2010 .

Avadhani, A; Tupkari, JV; Khambaty, A; Sardar, M. 2009. Cementum annulations and age determinations. Journal of Forensic Dental Sciences 1(2): $73-76$.

Brokx, PA. 1972. Age determination of venezuelan white-tailed deer. The Journal of Wildlife Management 36(4): 1060-1067.

Connolly, GE; Dudzinski, ML; Longhurst, WM. 1969. An improved age-lens weight regression for black-tailed deer and mule deer. The Journal of Wildlife Management 33(3): 701-704.

Cook, RL; Hart, RV. 1979. Ages assigned known age Texas white-tailed deer: tooth wear versus cementum analysis. Proceedings of the Annual Conference of the Southeastern Association of Fish and Wildlife Agencies 33: 195-201.

Erickson, JA; Anderson, AE; Medin, DE; Bowden, DC. 1970. Estimating ages of mule deer - an evaluation of technique accuracy. The Journal of Wildlife Management 34(3): 523531.

Erickson, JA; Seliger, WG. 1969. Efficient sectioning of incisors for estimating ages of mule deer. The Journal of Wildlife Management 33(2): 384-388.

Gasaway, WC; Harkness, DB; Rausch, RA. 1978. Accuracy of moose age determinations from incisor cementum Layers. The Journal of Wildlife Management 42(3): 558-563.

Gilbert, FF. 1966. Aging white-tailed deer by annuli in the cementum of the first incisor. The Journal of Wildlife Management 30(1): 200202.

Gilbert, FF; Stolt, SL. 1970. Variability in aging maine white-tailed deer by tooth-wear characteristics. The Journal of Wildlife Management 34(3): 532-535.

Hackett, EJ; Guynn, DC; Jacobson, HA. 1979. Differences in age structure of white-tailed deer in Mississippi produced by two aging-techniques. Proceedings of the Annual Conference of the Southeastern Association of Fish and Wildlife Agencies 33: 25 - 29.

Halls, LK. 1980. White-tailed deer. En: Big game of North America, ecology and management. Eds. JL Schmidt; DL. Gilbert. Wildlife Management Institute. Stackpole Books. Harrisburg, PA, US. Segunda impresión. p. 43 - 65.

Hamlin, KL; Pac, DF; Sime, CA; De Simone, RM; Dusek, GL. 2000. Evaluating the accuracy of ages obtained by two methods for Montana ungulates. The Journal of Wildlife Management 64(2): 441-449.

Jacobson, HA; Reiner, RJ. 1989. Estimating age of white-tailed deer: tooth wear versus cementum annuli. Proceedings of the Annual Conference of the Southeastern Association of Fish and Wildlife Agencies 43: 286-291. 
Kroll, J. 1994. A practical guide to producing and harvesting white-tailed deer. Institute for White-tailed Deer Management and Research. Center for Applied Studies in Forestry. College of Forestry. Stephen F. Austin State University. Nacogdoches, Texas, US. 591p.

Larson, JS; Taber, RD. 1987. Criterios de sexo y edad. En: Manual de técnicas de gestión de vida silvestre. 4 ed. en español. Ed. R Rodriguez Tarrés. Trad. B Orejas Miranda; A Fontes Riganti. The Wildlife Society. Maryland, US. p:151 $-164$.

Lockard, GR. 1972. Further studies of dental annuli for aging white tailed deer. The Journal of Wildlife Management 36(1): 46-55.

Low, WA; Cowan, IM. 1963. Age determination of deer by annular structure of dental cementum. The Journal of Wildlife Management 27(3): 466-471.

Maffei, L, Becerra, MN. 2000. Técnica básica para determinar la edad en ungulados silvestres en base al análisis de dientes. Ecología en Bolivia 34: 39 - 44.

Reimers, E. Nordby, Ø. 1968. Relationship between age and tooth cementum layers in Norwegian reindeer. The Journal of Wildlife Management 32(4): 957-961.

Rice, LA. 1980. Influences of irregular dental cementum layers on aging deer incisors. The Journal of Wildlife Management 44(1): 266-268.

Sauer, PR. 1984. Physical characteristics. En: White Tailed deer, ecology and management. Ed. LK Halls. The Wildlife Management Institute. Stackpole Books. Harrisburg, PA, US. p. $73-90$.

Sergeant, DE; Pimlott, DH. 1959. Age determination in moose from incisor teeth. The Journal of Wildlife Management 23(3): 315-321.

Severinghaus, CW. 1949. Tooth development and wear as a criteria of age in white-tailed deer. The Journal of Wildlife Management 13(2):195-215.

Taber, RD. 1960. Criteria of sex and age. En: Manual of Game Investigational Techniques.
Ed. HS Mosby. The Wildlife Society. Blacksburg, Virginia, US. p. 6:45 - 6:54.

Taber, RD. 1971. Criteria of sex and age. En: Wildlife Management Techniques. 3 ed. Rev. Ed. RH Giles Jr. The Wildlife Society. Washington DC, US. p. 379-390.

Thomas, DC; Bandy, PJ. 1973. Age determination of wild black tailed deer from dental annulations. The Journal of Wildlife Management 37(2): 232-235.

Vásquez, P; Burneo, F; Canziani, E; Ríos, J. 2007. Las plantas silvestres en la alimentación del venado cola blanca. Coto de Caza El Angolo-Piura. Guía de campo para su reconocimiento. Centro de Datos para la Conservación - Universidad Nacional Agraria La Molina. Lima, Perú. 154 p.

WHP (Wildlife Heritage Productions). 1984. Speedager (cartilla de determinación de edad para el venado de cola blanca con fotos que muestran el patrón de desgaste).

Presentado: 21/03/2011 Aceptado: 13/04/2017 